\title{
Differences in Cerebral Angioarchitectonics in Alzheimer's Disease in Comparison with Other Neurodegenerative and Ischemic Lesions
}

\author{
Ivan V. Maksimovich \\ Clinic of Cardiovascular Diseases Named after Most Holy John Tobolsky, Moscow, Russia \\ Email: carvasc@yandex.ru
}

How to cite this paper: Maksimovich, I.V. (2018) Differences in Cerebral Angioarchitectonics in Alzheimer's Disease in Comparison with Other Neurodegenerative and Ischemic Lesions. World Journal of Neuroscience, 8, 454-469. https://doi.org/10.4236/wjns.2018.84036

Received: October 6, 2018

Accepted: November 12, 2018

Published: November 15, 2018

Copyright $\odot 2018$ by author and Scientific Research Publishing Inc. This work is licensed under the Creative Commons Attribution International License (CC BY 4.0).

http://creativecommons.org/licenses/by/4.0/

\section{(c) (i) Open Access}

\begin{abstract}
Introduction: The research focuses on the clinical study of cerebral angioarchitectonics and microcirculation disorders in the development of Alzheimer's disease $(\mathrm{AD})$ in comparison with other neurodegenerative and ischemic lesions. Materials and methods: 1117 patients with different types and stages of neurodegenerative and ischemic lesions were examined, 93 of whom $(8.33 \%)$ had different stages of $\mathrm{AD}-$ Test Group; 1024 (91.67\%) had cerebral atherosclerosis, Binswanger disease (BD), vascular Parkinsonism (VP)-Control Group. The examination included definition of CDR, MMSE, cerebral CT, MRI, cerebral sciagraphy (SG), rheoencephalography (REG), morphometric detection of $\mathrm{AD}$ stages with TDR, and cerebral multi-gated angiography (MUGA). Results: In all patients with $\mathrm{AD}$, regardless of the disease stage, specific cerebral small vessel disease (CSVD), manifested by dyscirculatory angiopathy of Alzheimer's type (DAAT), was detected in the temporal and fronto-parietal areas. Conclusions: DAAT is an $\mathrm{AD}$-specific lesion of cerebral microvessels that changes hemodynamics, causes cerebral hypoxia, and contributes to impaired amyloid beta metabolism. The combination of deposition of amyloid beta in the cerebral tissue and vascular wall, as well as specific disorders of microcirculation, cause neurodegeneration and AD development. Patients with other neurodegenerative and ischemic lesions had no DAAT manifestations.
\end{abstract}

\section{Keywords}

Alzheimer's Disease, Dementia, CSVD, Cerebral Atrophy, Tomography Dementia Rating Scale, TDR, Dyscirculatory Angiopathy of Alzheimer's Type, DAAT

\section{Introduction}

Cerebral ischemic and neurodegenerative diseases are increasingly observed 
among the population of different countries [1]. The most common of them are atherosclero sclerosis of the brain, Alzheimer's disease (AD), Binswanger's disease (BD), and Parkinsonism. All these diseases lead to the progression of dementia and cognitive disorders and are also accompanied by the development of cerebral small vessel disease (CSVD) [2] [3] [4]. This condition is caused by the peculiarity of the microcirculation in the brain, since there are about 3 - 4 thousand capillaries in one cubic centimeter of the cerebral tissue. With such a high demand for blood supply, disorders in hemoperfusion and hemodynamics cause or contribute to the development of neurodegenerative processes [5].

In the early stages, clinical pictures of these diseases have much in common.

$\mathrm{AD}$ is one the leading neurodegenerative diseases nowadays. Currently, there are over 36 million people suffering from $\mathrm{AD}$ [1] [4]. In the USA, this disease affects more than 5 million people, while $5 \%$ of them acquire $\mathrm{AD}$ at a fairly young age [1].

$\mathrm{AD}$ begins to develop in secret, years or even decades before the primary manifestations of the disease [6]. The preclinical stage of $\mathrm{AD}$, in which the patient does not make any classical complaints, is difficult to detect [1]. Since this disease is often hereditary, it is necessary to examine AD patients' relatives and descendants for the detection of its preclinical stage [1]. It shows that the true number of patients suffering from $\mathrm{AD}$ can be much greater [1] [6].

$\mathrm{AD}$ is accompanied by the development of involutive changes in the hippocampus and temporal cerebral lobes, but the etiology and pathogenesis of this disease have not been fully studied [7]. In recent years, the use of CT, MRI, PET, the introduction of biomarkers, and research on biological models have made it possible to achieve serious results in the study of structural and morphological changes taking place in the brain tissue during this disease, as well as to achieve success in understanding the distribution of amyloid beta and tau [6] [8]-[14]. However, the dependence of cerebral involutive changes and the disorders in cerebral angioarchitectonics and microcirculation is not completely clear [15] [16] [17] [18] [19].

Cerebral small vessel disease (CSVD) and cerebral hypoperfusion are important in the development of AD [3] [17] [19]-[24]. These processes are specific and connected with disorders in capillary blood supply [17] [19] [20] [21]. Patients undergo a change in the distal arterial and capillary bed, which leads to damaging the neurovascular unit (NVU) in the form of reduction in the number of microvessels and damaging tissue elements [17] [18] [22].

These changes lead to neuronal degeneration, a decrease in the clearance and an increase in the accumulation of amyloid causing further neurovascular dysfunction and neurodegeneration [18] [21] [25] [26] [27]. Beta amyloid deposits in the cerebral tissue and especially in the vascular wall reduce the elasticity of microvessels and cause the narrowing of their lumen reducing in a higher degree the cerebral blood flow, which in its turn causes more active amyloid deposition and accelerates AD development [28].

There is no complete clinical picture of all CSVD components involved in the 
development of $\mathrm{AD}$. Besides, it is not fully clear how exactly cerebrovascular dysfunction affects neurodegenerative processes and the development of atrophic changes in the brain. Moreover, there is a question of when these changes appear before the primary clinical manifestations of AD [20] [29].

The present research is devoted to clinical identification of changes in cerebral angioarchitectonics and microcirculation in $\mathrm{AD}$, to determining the relationship of these changes with cerebral involutive changes, and to comparing these changes with vascular and involutive changes that occur in other neurodegenerative and ischemic cerebral diseases.

Consequently, the research includes patients suffering from various cerebral neurodegenerative and ischemic lesions: Alzheimer's disease (AD), various types of cerebral atherosclerosis, Binswanger's disease (BD) and vascular Parkinsonism (VP), who have been under examination in our clinic for the recent 15 years.

\section{Materials and Methods}

\subsection{Patient Selection}

All the research described in this paper was accomplished with the approbation of the Ethics Committee and with the agreement of the examined patients and their relatives.

We selected 1117 patients who were 34 - 81 years old, mean age being 75.5 of whom men comprised $792(70.90 \%)$ cases and women 325 (29.10\%) cases. Those patients had primary signs of cerebral neurodegeneration or suffered from marked neurodegenerative lesions of the brain.

\subsection{Patient Examination}

Examination plan:

- dementia severity was clinically assessed in accordance with the Clinical Dementia Rating scale (CDR) [30];

- cognitive functions were evaluated with the help of Mini-Mental State Examination (MMSE) [31];

- coagulogical, biochemical and general clinical indicators were measured by laboratory tests;

- brain scintigraphy (SG), with TC $99 \mathrm{M}$ pertechnetate 555, was executed using a gammacamera (Ohio Nuclear Company, USA) and following the classical method in dynamic and static modes;

- rheoencephalography (REG) was accomplished in standard automatic modes determining disorders in pulse blood volume in the cerebral hemispheres using "Reospektr-8" (Neurosoft Company, Russia);

- CT and MRI of the brain were conducted by means of "Somatom" (Siemens), "Hi Speed" (GE), "Tomoscan" (Philips), "Apetro Eterna" (Hitachi). The ATAA (Advance Tomo Area Analysis) [23] [24] was used during the research allowing the determination of the percentage of the temporal lobes tissue volume decrease in comparison with their natural volume, showing 
thus the cerebral temporal atrophic changes [29];

- morphometrical assessment of dementia severity and of AD stages was made following Tomography Dementia Rating scale (TDR) [32]. The method allows the determination of dementia stages during $\mathrm{AD}$ taking into account the severity of atrophic changes of the temporal lobes shown by CT and MRI. The essence of the method is the following. During CT or MRI, using the Advance Tomo Area Analysis computer program (ATAA) [24], the area of the middle cranial fossa is measured successively on each scan. The automatically obtained data are recalculated by the thickness of the scan, then the scans are summed and the volume of the middle cranial fossa is determined corresponding to the normal volume of the cerebral tissue of the temporal regions [29]. Simultaneously, the percentage of tissue loss in the cerebral temporal lobes is calculated in each case [17]. Next, the obtained reduced volumes of temporal lobes are compared with the clinical picture of dementia on the CDR scale, which results in an objective, morphometrically validated assessment of the stage of dementia in $\mathrm{AD}$ [32];

- cerebral multi-gated angiography (MUGA) was executed using "Advantx" (GE) by transfemoral access following the classical method of introducing Omnipack 350, $10-12 \mathrm{ml}$ intracarotidally and $7-8 \mathrm{ml}$ intravertebrally. The procedure was conducted in front and side projections, at the registration speed of 25 shots per second and in the mode of constant subtraction [17] [19]. Capillary blood flow was measured by Angio Vision computer program [23] which allows to define the changes in the density and the number of black pixels in the corresponding area of the cerebral angiographic image as radiopaque substance passes through arterioles and capillaries [17] [19]. Recently, a similar program with the addition of 2D effects has been proposed by Philips [33].

\subsubsection{Test Group}

$93(8.33 \%)$ patients of 34 - 79 years old, mean age 67: 32 men (34.40\%) and 61 (65.59\%) women. They had different AD stages.

\subsubsection{Control Group}

1024 (91.67\%) patients of 28 - 81 years old, mean age 73: 594 (58.01\%) men and 430 (41.99\%) women. They had neurodegenerative lesions of different etiology without extensive ischemic foci. The lesions were accompanied by cerebral involutive changes, symptoms of dementia and cognitive disorders comparable in their severity with disorders in Test Group patients. According to the etiology of the disease and its severity, all patients were divided into the following groups:

- 27 (2.64\%) people had initial signs of chronic cerebrovascular insufficiency of atherosclerotic genesis. Patients of this group had complaints that indicated disorders in cerebral hemodynamics;

- 577 (56.35\%) patients had marked signs of chronic cerebrovascular insufficiency of atherosclerotic genesis, 198 (34.32\%) of them had had transient cerebral circulation disorders; 
- $342(33.40 \%)$ patients had a severe form of chronic cerebrovascular insufficiency accompanied by multiple atherosclerotic lesions of the brain. In the anamnesis, they had small focal single or multiple strokes;

- $23(2.25 \%)$ patients suffered from Binswanger's disease (BD);

- 55 (5.37\%) patients suffered from vascular Parkinsonism (VP);

The number of patients in the control group is determined by the need to compare vascular, microcirculatory and atrophic changes in the brain in the above-mentioned nosology to specific changes found among test group patients.

\section{Results}

\subsection{Test Group}

In accordance with CT and MRI data, we identified specific, morphometrically validated, atrophic changes in the hippocampus and temporal lobes corresponding to different $\mathrm{AD}$ stages with a certain level of dementia. Thanks to these data and the developed classification called Tomography Dementia Rating scale (TDR) [32], the patients were divided:

- at the time of this research, $10(10.75 \%)$ patients had preclinical AD stage TDR- 0 characterized by the absence of dementia. However, the patients had increasing memory disorders. Cognitive functions were reduced to $26-28$ MMSE points, initial involutive changes in the brain were manifested by the atrophy of the temporal lobes with a $4 \%-8 \%$ decrease in the tissue mass. Each of these patients had direct relatives suffering from AD (Figure 1(A)), (Table 1);

- at the time of this research, 26 (27.96\%) patients had early AD stage TDR-1 corresponding to mild dementia. Their cognitive functions were reduced to 20 - 25 MMSE points, dementia was at the level of CDR-1, involutive changes in the brain were manifested by the atrophy of the temporal lobes with a $9 \%-$ $18 \%$ decrease in the tissue mass. The anamnesis of the disease was 2 years (Figure 1(B)), (Table 1);

- at the time of this research, 40 (43.01\%) patients had middle AD stage TDR-2 corresponding to moderate dementia. Cognitive functions were reduced to 12 - 19 MMSE points; dementia was at CDR-2 level. Involutive changes in the brain were manifested by the atrophy of the temporal lobes with a $19 \%$ $32 \%$ decrease in the tissue mass. The anamnesis of the disease was $2-6$ years (Figure 1(C)), (Table 1);

- at the time of this research, 17 (18.28\%) patients had late AD stage TDR-3 due to severe dementia. Cognitive functions were reduced to 7 - 11 MMSE points, dementia was at the level of CDR-3, involutive changes in the brain were manifested by the atrophy of the temporal lobes with a $33 \%-62 \%$ decrease in the tissue mass. The anamnesis of the disease was $7-11$ years (Figure 1(D)), (Table 1).

The general character of the cerebral involutive changes is presented in Table 1. 

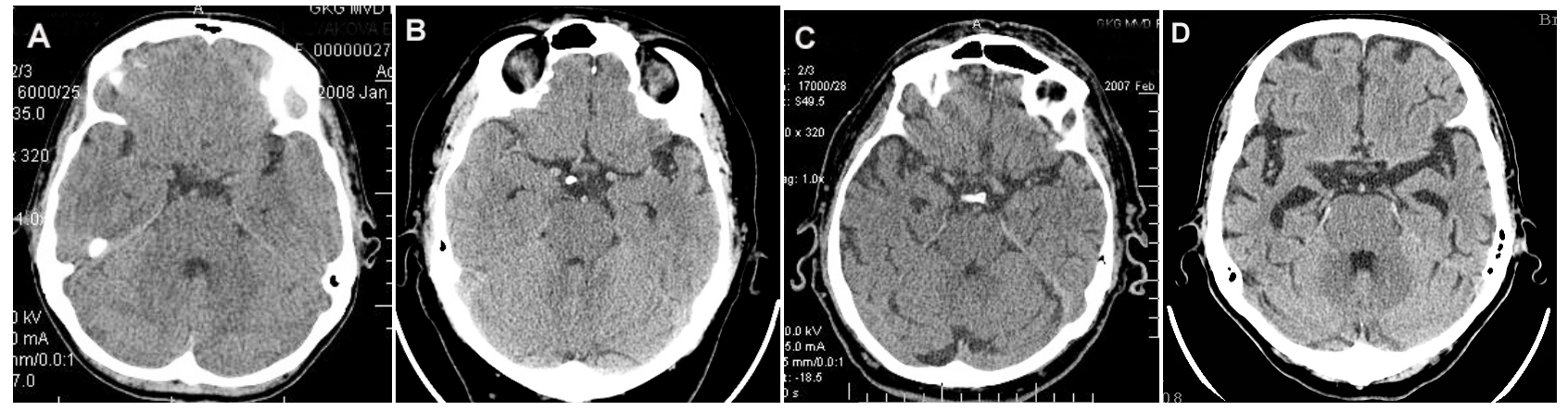

Figure 1. Brain CT of patients with various AD stages. (A) pre-clinical stage TDR-0 (tissue mass decrease: right temporal lobe-6\%, left temporal lobe-8\%); (B) early stage TDR-1 (tissue mass decrease: right temporal lobe-9\%, left temporal lobe-14\%); (C) middle stage TDR-2 (tissue mass decrease: right temporal lobe-27\%, left temporal lobe-20\%); (D) late stage TDR-3 (tissue mass decrease: right temporal lobe-42\%, left temporal lobe-38\%).

Table 1. Cerebral changes in examined patients (CT and MRI data).

\begin{tabular}{|c|c|c|c|}
\hline CEREBRAL CHANGES & $\begin{array}{l}\text { Test Group } \\
\text { N-93 }\end{array}$ & $\begin{array}{c}\text { Control Group } \\
\mathrm{N}-1012\end{array}$ & p (chi-square) \\
\hline \multicolumn{4}{|l|}{ Changes in temporal areas } \\
\hline Local involutive changes of the brain cortex in temporal areas & 93 & 0 & \\
\hline Local atrophy of temporal lobes with $4 \%-8 \%$ decrease in tissue mass (TDR-0) & 10 & 0 & \\
\hline Local atrophy of temporal lobes with $9 \%-18 \%$ decrease in tissue mass (TDR-1) & 26 & 0 & $\mathrm{p}<0.01$ \\
\hline Local atrophy of temporal lobes with $19 \%-32 \%$ decrease in tissue mass (TDR-2) & 40 & 0 & \\
\hline Local atrophy of temporal lobes with $33 \%-62 \%$ decrease in tissue mass (TDR-3) & 17 & 0 & \\
\hline \multicolumn{4}{|l|}{ General cerebral changes } \\
\hline Multiple calcium salts deposits in intracranial vessels & 0 & 998 & $\mathrm{p}<0.01$ \\
\hline Single and multiple post-ischemic microcysts (more than $5 \mathrm{~mm}$ ) & 0 & 132 & $\mathrm{p}<0.01$ \\
\hline Post-ischemic microcysts of less than $5 \mathrm{~mm}$ & 0 & 148 & $\mathrm{p}<0.01$ \\
\hline General neurodegenerative changes in the cortex of the brain & 48 & 876 & $\mathrm{p}<0.01$ \\
\hline Sylvian fissures widening signs & 93 & 974 & Not significant \\
\hline Leucoaraiosis signs & 0 & 178 & $\mathrm{p}<0.01$ \\
\hline Unocclusive hydrocephaly signs & 57 & 453 & $\mathrm{p}<0.01$ \\
\hline
\end{tabular}

To define the differences of the symptoms under study, we conducted an analysis of contingency tables with Chi-square criterion. All the figures except for "Sylvian fissures widening signs" revealed significant differences $(\mathrm{p}<0.01)$. The statistical analysis was accomplished with the help of Statsoft Statistica 10 program.

According to laboratory tests:

- 35 (37.63\%) patients had increased blood lipids level.

- 39 (41.94\%) patients had hypercoagulation.

SG showed that all $93(100 \%)$ patients had the slowing of blood flow in the brain hemispheres.

REG demonstrated that 93 (100\%) patients had a decrease in volumetric pulse blood supply in the carotid system.

MUGA revealed (Table 2) the following vascular and microvascular disord- 
ers:

- there were no atherosclerotic changes of extra- and intracranial arteries in 84 (90.32\%) patients, and 9 (9.68\%) patients had a minor degree of them;

- all $93(100 \%)$ patients had a decline in capillary contrasting caused by a decrease of the number of capillaries locally in the temporal and fronto-parietal areas in the form of hypervascular zones (Figure 2(A1), Figure 2(D1));

- all $93(100 \%)$ patients featured multiple arteriovenous shunts in the basin of the anterior ciliary arteries supplying blood locally for the temporal lobes, as well as in the basin of distal arterial branches supplying blood for fronto-parietal areas of the brain (Figure 2(A2), Figure 2(B2), Figure 2(C2)). It is through these shunts that the early venous discharge of arterial blood occurs, along with the simultaneous contrasting of arteries and veins (Figure 2(B3);

- $84(90.32 \%)$ patients had local stagnation of venous blood, abnormally widened laterally located venous trunks which receive blood from arteriovenous shunts of the temporal and fronto-parietal areas (Figure 2(C4));

- 85 (91.40\%) patients had stagnation of venous blood at the border between the frontal and parietal areas caused by increased blood flow from arteriovenous shunts (Figure 2(C5));

- $74(79.57 \%)$ patients featured increased looping of distal intracranial arterial branches (Figure 2(D6)).

We named the combination of these changes in cerebral microcirculation and Angioarchitectonics, which are specific for $\mathrm{AD}$ and which lead to dysregulation of blood flow, “dyscirculatory angiopathy of Alzheimer's type" (DAAT) [17] [19] [29].
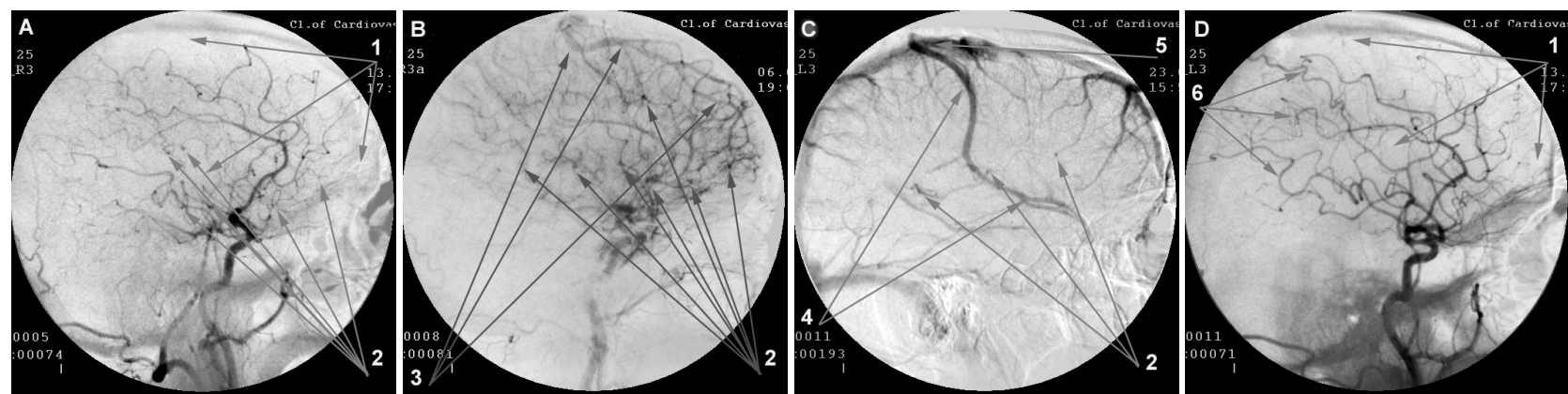

Figure 2. Dyscirculatory angiopathy of Alzheimer's type (DAAT) at AD different stages. (A) Right internal carotid artery angiogram, arterial phase: 1 reduction of the number of capillaries in the temporal and fronto-parietal area; 2 multiple arteriovenous shunts in the temporal and fronto-parietal area; (B) Right internal carotid artery angiogram, capillary phase: 2 multiple arteriovenous shunts in the temporal and fronto-parietal area; 3 early discharge of arterial blood through arteriovenous shunts into the venous bed with simultaneous filling of arteries and veins; (C) Left internal carotid artery angiogram, venous phase: 2 multiple arteriovenous shunts in the temporal and fronto-parietal area; 4 Development of abnormally widened lateral venous trunks; 5 stagnation of venous blood at the border of the frontal and parietal area; (D) Right internal carotid artery angiogram, arterial phase: 1 reduction of the number of capillaries in the temporal and fronto-parietal area; 6 increased looping of distal intracranial arterial branches. 
Table 2. Cerebral Angioarchitectonics and microcirculation features in the examined patients.

\begin{tabular}{|c|c|c|c|}
\hline CEREBRAL VASCULAR CHANGES & $\begin{array}{c}\text { Test Group } \\
\text { N-93 }\end{array}$ & $\begin{array}{l}\text { Control Group } \\
\mathrm{N}-1012\end{array}$ & p (Chi-square) \\
\hline Atherosclerotic changes of intracranial arteries & 0 & 989 & $\mathrm{p}<0.01$ \\
\hline Stenotic lesions of intracranial branches & 0 & 815 & $\mathrm{p}<0.01$ \\
\hline Occlusive lesions of intracranial arterial branches & 0 & 174 & $\mathrm{p}<0.01$ \\
\hline Local reduction of capillary blood flow in temporal and fronto-parietal areas & 93 & 0 & $\mathrm{p}<0.01$ \\
\hline Local multiple arteriovenous shunts in temporal and fronto-parietal areas & 93 & 0 & $\mathrm{p}<0.01$ \\
\hline $\begin{array}{l}\text { Local early discharge of arterial blood into the venous bed in temporal and } \\
\text { fronto-parietal area }\end{array}$ & 93 & 0 & $\mathrm{p}<0.01$ \\
\hline $\begin{array}{l}\text { Local development of abnormally widened lateral venous branches in temporal and } \\
\text { fronto-parietal areas }\end{array}$ & 84 & 0 & $\mathrm{p}<0.01$ \\
\hline Abnormal stagnation of venous blood at the border of frontal and parietal areas & 85 & 0 & $\mathrm{p}<0.01$ \\
\hline Increased looping of distal intracranial branches & 74 & 68 & $\mathrm{p}<0.01$ \\
\hline $\begin{array}{l}\text { Separate, subcortical disseminated parts of reduction of capillary blood flow at the level } \\
\text { of the white matter of the brain }\end{array}$ & 0 & 415 & $\mathrm{p}<0.01$ \\
\hline $\begin{array}{l}\text { Multiple subcortical disseminated arteriovenous shunts at the level of the white matter } \\
\text { of the brain }\end{array}$ & 0 & 408 & $\mathrm{p}<0.01$ \\
\hline $\begin{array}{l}\text { Disseminated, subcortical, early discharge of arterial blood into the venous bed at the } \\
\text { level of the white matter of the brain }\end{array}$ & 0 & 388 & $\mathrm{p}<0.01$ \\
\hline
\end{tabular}

To define the differences in the symptoms under study, we conducted an analysis of contingency tables with Chi-square criterion. All the figures revealed significant differences $(\mathrm{p}<0.01)$. The statistical analysis was accomplished with the help of Statsoft Statistica 10 program.

\subsection{Control Group}

According to $\mathrm{CT}$ and MRI:

- No isolated atrophic changes of the hippocampus and temporal lobes of the brain specific for AD were detected in any case (Table 1);

- There were general cerebral involutive and neurodegenerative changes of different localization and severity in all 1024 cases (100\%) (Table 1).

In determining the clinical stage of dementia (CDR) and the severity of cognitive disorders (MMSE), it was revealed:

- 27 patients with initial signs of chronic cerebrovascular insufficiency of atherosclerotic genesis showed no clear signs of dementia or cognitive disorders;

- 577 patients with severe chronic cerebrovascular insufficiency of atherosclerotic origin featured dementia: CDR-1 level was detected in 262 (45.41\%) patients, CDR-2 level-in 315 (54.59\%) patients, which was accompanied by a decrease in cognitive functions to 18 - 25 MMSE points in all patients;

- 342 patients with severe chronic cerebrovascular insufficiency demonstrated dementia: CDR-2 level was detected in 253 (73.98\%) patients, CDR-3 level-in $89(8.79 \%)$ patients, which was accompanied by a decrease in cognitive functions to 11 - 19 MMSE points in all patients;

- 23 patients with Binswanger's disease had dementia: CDR-1 level was detected in 16 (69.57\%) patients, CDR-2 level-in 7 (30.43\%) patients, which 
was accompanied by a decrease in cognitive functions to 12 - 25 MMSE points in all patients;

- 55 patients with vascular Parkinsonism had dementia: CDR-1 level was detected in 29 (52.73\%) patients, CDR-2 level-in 26 (47.27\%) patients, which was accompanied by a decrease in cognitive functions to 18 - 25 MMSE points in all patients.

According to laboratory tests:

- $809(79.00 \%)$ patients had increased blood lipids level.

- $769(75.10 \%)$ patients had hypercoagulation.

SG revealed the slowing down of blood flow in the hemispheres in all 1024 (100\%) patients.

REG demonstrated a decline in volume pulse blood supply in the carotid system in all $1024(100 \%)$ patients.

Cerebral MUGA revealed the following disorders (Table 2):

- signs of atherosclerotic changes of the intracerebral arterial bed-993 (96.97\%) patients (Figure 3(A1), Figure 3(A2), Figure 3(B1), Figure 3(B3));

- stenotic changes of intracerebral arterial branches-824 (80.47\%) patients (Figure 3(A2), Figure 3(B1), Figure 3(B3));

- occlusions of intracerebral arterial branches-178 (17.38\%) patients (Figure 3(A1), Figure 3(B3));

- no cases of AD-specific local reduction of the capillary bed with characteristic borders in the projection of the temporal and fronto-parietal areas of the brain;

- no cases of $\mathrm{AD}$-specific multiple local arteriovenous shunts in the basins of arterial branches supplying blood for the temporal and fronto-parietal areas of the brain;

- certain subcortical, disseminated at the level of the white matter in various parts of the brain, areas of lowered capillary contrast-419 (40.92\%) patients (Figure 3(C1));

- multiple subcortical disseminated arteriovenous shunts within the limits of the white matter of the brain-413 (40.33\%) patients (Figure 3(C2), Figure 3(D1));

- subcortical, disseminated, early discharge of arterial blood into the venous bed at the level of the white matter of the brain-392 (38.28\%) patients;

- no cases of $\mathrm{AD}$-specific, abnormally widened, laterally located venous trunks at the level of fronto-parietal areas;

- no cases of $\mathrm{AD}$-specific stagnation of venous blood at the border of the frontal and parietal area;

- development of increased looping of distal intracranial arterial branches-69 (6.74\%) patients (Figure 3(B2)).

Thus, the obtained data suggest that the abovementioned changes identified in Control Group patients are completely different from the changes detected in Test Group patients. 

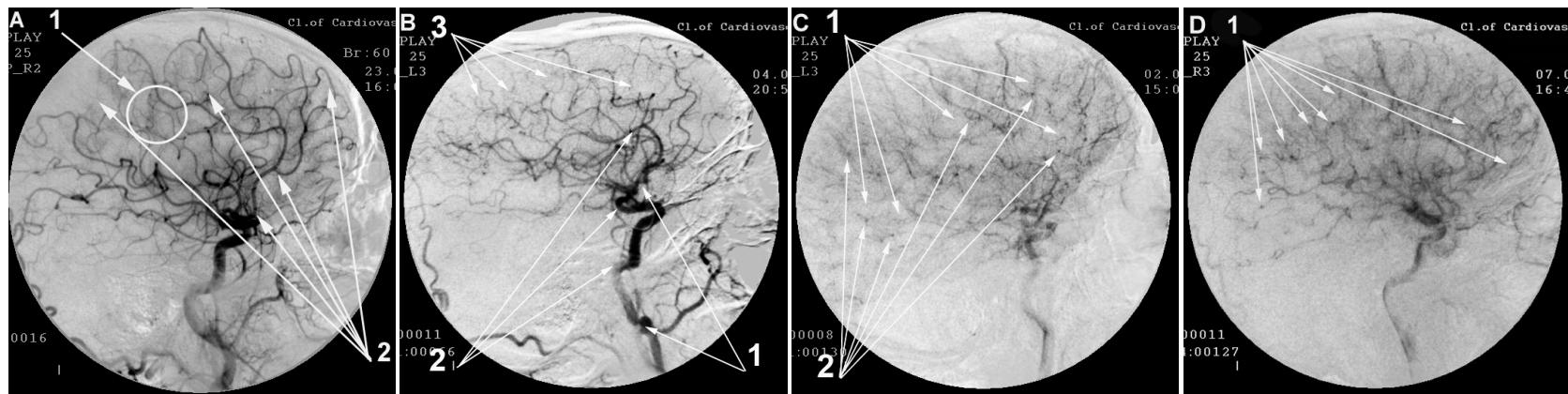

Figure 3. Changes in the distal arterial and microcirculatory intracerebral bed in control group patients. (A) Angiogram of the right internal carotid artery, arterial phase: 1 Multiple occlusions of the distal branches of the middle cerebral artery; 2 Multiple atherosclerotic stenotic changes in intracerebral arteries; (B) Angiogram of the left internal carotid artery, arterial phase: 1 Multiple atherosclerotic stenotic changes in the proximal parts of the cerebral arteries; 2 Increased tortuosity of the cerebral arterial branches; 3 Multiple stenotic and occlusive lesions of distal intracerebral arterial branches; (C) Angiogram of the left internal carotid artery, capillary phase: 1 Depletion of the capillary bed at the level of the white matter of the brain; 2 Multiple small arteriovenous shunts at the level of the white matter of the brain; (D) Angiogram of the right internal carotid artery, capillary phase: 1 Multiple small arteriovenous shunts at the level of the white matter of the brain.

\section{Discussion}

Research aimed at studying vascular and microcirculatory disorders in AD has been basically conducted using post-mortem autopsies [20] [21] or genetically modified mice having an experimental AD-model [4] [18] [25]. The research described here was carried out in clinical settings.

The patients of the test and control groups are comparable by the severity of dementia according to CDR, the level of cognitive impairment according to MMSE and the severity of the general condition. However, vascular and microcirculatory disorders, as well as atrophic cerebral changes in these groups are of a completely different character.

The data obtained in this research show that intracerebral vascular and microcirculatory changes in $\mathrm{AD}$ have a pronounced specificity in Test Group patients and, therefore, these changes are called the dyscirculatory angiopathy of Alzheimer's type (DAAT). The combination of these changes is an important factor in the development and diagnosis of the disease.

Test Group patients, regardless of their age, practically do not have atherosclerotic changes in intracerebral vessels, and they do not feature stenotic and occlusive lesions of arteries, arterioles and capillaries.

All 93 (100\%) Test Group patients showed a decrease in the number of microvessels and depletion of the capillary bed, which leads to the development of hypovascular zones in the temporal and fronto-parietal areas.

The blood flowing through larger arterial branches cannot get through reduced arterioles and capillaries, which leads to arteriovenous shunts in the corresponding cerebral regions making hypoxia worse.

Arteriovenous shunts are a natural defensive reaction of the organism against the failure of distal blood to flow smoothly through the capillary bed [5] [17] [19]. Due to these shunts, arterial blood is dumped into the venous bed. 
A large flow of blood from these arteriovenous shunts leads to the development of large venous trunks that dump blood into the venous sinus, which in turn causes blood stagnation at the level of the fronto-parietal area.

As $\mathrm{AD}$ develops, cerebral angiogenesis declines; acquired cerebral vascular changes lead to increased curving of distal arteries and capillaries [17] [19] [34].

Our findings also explain the results of research of many authors who study microcirculatory changes in $\mathrm{AD}$ on the materials of post mortem autopsy and experimental animals and show that the number of capillaries in the tissue of the hippocampus and the limbic system decreases [3] [4] [18] [20]. Besides, our data confirm the studies which point to the thinning of the capillaries, the reduction of their further branching [21] [27] [34] [35] [36], the reduction of the distal arterial influx and the development of cerebral hypoperfusion and hypoxia [35] [37]-[43].

DAAT begins to develop decades before possible $\mathrm{AD}$ development and is probably of a congenital nature [17] [36]. The signs of these changes are detected in the childhood and at the pre-clinical stage of the disease (TDR-0) of Test Group patients. It is obvious that DAAT features at a young age are not a guarantee of $\mathrm{AD}$ development, but it is a predictor of the possible development of this disease. The organism may compensate these disorders for a long time, which prevents $\mathrm{AD}$ development. At a certain stage compensatory abilities of the organism lessen, progressive disorders in amyloid metabolism occur in cerebral tissues and the vessel wall, and as a result, the process transfers into an active development stage of the disease. The process develops along with age [41] [44] [45].

As it has been noted in many works, these microvascular disorders cause neurovascular dysfunction [22] [38] [43], metabolic disorders [34] [35], and the development of hypoxia and hypometabolism in the cerebral tissue [39] [41]. Simultaneously, there occurs degeneration and neuronal death, synaptic loss, loss of mitochondria in the cells of the smooth endoplasmic reticulum and Golgi apparatus and general neurodegeneration [21] [22] [34] [41] [42]. In turn, the reduction of capillary blood inflow and venous outflow disorders lead to a decrease in the process of amyloid beta elimination and to an increase in its accumulation in cerebral tissues and vessel walls [4] [18] [25]. The combination of these processes further violates cerebral microcirculation and leads to aggravation of the patient's condition [3] [28] [43]. This process, in turn, is accompanied by a dysfunction of the blood-brain barrier (BBB) [25] [45] [46].

The more pronounced these disorders are, the more likely the development and progression of $\mathrm{AD}$ are [19] [27] [32] [34] [35] [42] [47].

Initial cerebral involutive and hypotrophic changes manifested in a $4 \%-8 \%$ decrease in temporal lobes tissue mass, were found in Test Group patients with preclinical AD stage (TDR-0). The data obtained indicate long stability of this condition that does not lead to the development of dementia and cognitive disorders. Further progression of atrophic changes in the temporal lobes promotes 
dementia and cognitive impairment and leads to the worsening of the patients' condition and $\mathrm{AD}$ development. Thus, the early AD stage (TDR-1) features a 9\% - $18 \%$ decrease of the temporal lobes tissue mass, middle $\mathrm{AD}$ stage (TDR-2)-19\% - 32\%, and late AD stage (TDR-3)-33\% - 62\%. At the same time, general atrophic changes in the brain develop in the late stages of the disease.

Control Group patients with such nosologies as atherosclerotic lesions, Binswanger's disease, and vascular Parkinsonism show no AD-specific changes in the cerebral vascular system and CSVD, as well as no specific cerebral atrophic changes similar to those of Test Group patients.

Control Group patients showed disseminated atherosclerotic changes accompanied by stenotic and occlusive lesions of intracerebral arteries and capillaries. This process is accompanied by the formation of disseminated arteriovenous shunts in the corresponding cerebral regions, as well as by the development of local zones of ischemia and gliosis in the cerebral tissue. In these patients, arteriovenous discharge of blood is less pronounced than in $\mathrm{AD}$.

Thus, with cerebral atherosclerosis, stenotic and occlusive lesions develop in the arteries and capillaries at the level of both gray and white matter of the brain, which is accompanied by the opening of small arteriovenous shunts in the same areas. However, the opening of these arteriovenous shunts does not usually lead to the development of large venous trunks or to a marked discharge of blood into the venous bed. At the same time, the hypotrophic and atrophic changes in the cerebral tissue are correspondingly widespread, which is confirmed by the results of studies by many authors [5] [48] [49].

Patients with $\mathrm{BD}$ feature the disseminated lesions of the distal subcortical arterioles and capillaries at the white matter level of the brain. The opening arteriovenous shunts are usually small in size, and also have a disseminated character. Obviously, the volume of blood discharged through them is not large, which does not lead to the development of large venous trunks and overflow of venous sinuses. Involutive and atrophic changes in the cerebral tissue also have a disseminated subcartical character. The data obtained by us are confirmed by studies carried out earlier [50] [51] [52] [53].

Patients with VP have a similar pattern, the difference being that arterioles and capillaries are affected in the thalamus, basal ganglia and bridge. Small arteriovenous shunts have a corresponding localization. Involutive changes are usually less pronounced and of limited character [34].

Involutive and hypotrophic changes in the brain in Control Group patients develop according to the ischemic, often disseminated type; they differ from the involutive changes occurring in Test Group patients with $\mathrm{AD}$ and are usually associated with the zones of cerebral ischemia.

\section{Conclusions}

Cerebral vascular and microcirculatory disorders in $\mathrm{AD}$ represent dyscirculatory 
angiopathy of Alzheimer's type (DAAT). These specific changes lead to the development of hypoxia, cause intercellular disorders, contribute to disorders in the metabolism of amyloid beta and to involutive changes in the temporal regions. The combination of these changes is a distinctive feature of $\mathrm{AD}$ in comparison with other ischemic and neurodegenerative diseases.

Vascular and microcirculatory changes in Control Group patients are of different genesis and do not lead to the development of DAAT. These changes are different, and often atherosclerotic, and lead to the development of other cerebral ischemic and neurodegenerative lesions.

\section{Funding}

The authors received no financial support for the research, authorship, and/or publication of this article.

\section{Conflicts of Interest}

The author declared no potential conflicts of interest with respect to the research, authorship, and/or publication of this article.

\section{References}

[1] Alzheimer's Association (2017) Alzheimer's Disease Facts and Figures. Alzheimer's \& Dementia 13, 325-373. https://doi.org/10.1016/j.jalz.2017.02.001

[2] Pantoni, L. (2010) Cerebral Small Vessel Disease: From Pathogenesis and Clinical Characteristics to Therapeutic Challenges. The Lancet Neurology, 9, 689-701. https://doi.org/10.1016/S1474-4422(10)70104-6

[3] Cai, Z., Wang, C., He, W., et al. (2015) Cerebral Small Vessel Disease and Alzheimer's Disease. Clinical Interventions in Aging, 23, 1695-1704. https://doi.org/10.2147/CIA.S90871

[4] Grammas, P., Martinez, J., Sanchez, A., et al. (2014) A New Paradigm for the Treatment of Alzheimer's Disease: Targeting Vascular Activation. Journal of Alzheimer's Disease, 40, 619-630. https://doi.org/10.3233/JAD-2014-132057

[5] Gjulev, N.M., Pustozertsev, V.G. and Gjulev, S.N. (2002) Cerebrovascular Diseases. Binom, Moscow.

[6] Mormino, E.C., Papp, K.V., Rentz, D.M., et al. (2017) Early and Late Change on the Preclinical Alzheimer's Cognitive Composite in Clinically Normal Older Individuals with elevated Amyloid $\beta$. Alzheimer's \& Dementia, 13, 1004-1012. https://doi.org/10.1016/j.jalz.2017.01.018

[7] Jack, C.R., Petersen, R.C, Xu, Y.C., et al. (1999) Prediction of AD with MRI-Based Hippocampal Volume in Mild Cognitive Impairment. Neurology, 52, 1397-1403. https://doi.org/10.1212/WNL.52.7.1397

[8] Waldemar, G., Dubois, B., Emre, M., et al. (2007) Recommendations for the Diagnosis and Management of Alzheimer's Disease and Other Disorders Associated with Dementia: EFNS Guideline. European Journal of Neurology, 14, e1-e26. https://doi.org/10.1111/j.1468-1331.2006.01605.x

[9] Burton, E.J., Barber, R., Mukaetova-Ladinska, E.B., et al. (2009) Medial Temporal Lobe Atrophy on MRI Differentiates Alzheimer's Disease from Dementia with Lewy Bodies and Vascular Cognitive Impairment: A Prospective Study with Pathological 
Verification of Diagnosis. Brain, 132, 195-203.

https://doi.org/10.1093/brain/awn298

[10] Trojanowski, J.Q., Vandeerstichele, H., Korecka, M., et al. (2010) Update on the Biomarker Core of the Alzheimer's Disease Neuroimaging Initiative Subjects. Alzheimer's \& Dementia, 6, 230-238. https://doi.org/10.1016/j.jalz.2010.03.008

[11] Adriaanse, A., Sanz-Arigita, E., Binnewijzend, M., et al. (2011) Molecular Markers of Alzheimer's Disease Pathology and Their Relationship with Default Mode Network Integrity. Alzheimer's \& Dementia, 7, S2-S3. https://doi.org/10.1016/j.jalz.2011.05.005

[12] Meyer, P.T., Hellwig, S., Amtage, F., et al. (2011) Dual-Biomarker Imaging of Regional Cerebral Amyloid Load and Neuronal Activity in Dementia with PET and ${ }^{11}$ C-Labeled Pittsburgh Compound B. Journal of Nuclear Medicine, 52, 393-400. https://doi.org/10.2967/jnumed.110.083683

[13] Weiner, W.W., Veitch, D.P., Aisen, P.S., et al. (2015) 2014 Update of the Alzheimer's Disease Neuroimaging Initiative: A Review of Papers Published since Its Inception. Alzheimer's \& Dementia, 11, e1-e120.

https://doi.org/10.1016/j.jalz.2014.11.001

[14] Chiang, G.C., Insel, P.S., Tosun, D., et al. (2011) Identifying Cognitively Healthy Elderly Individuals with Subsequent Memory Decline by Using Automated MR Temporoparietal Volumes. Radiology, 259, 844-851. https://doi.org/10.1148/radiol.11101637

[15] De la Torre, J.C. (1997) Hemodynamic Consequences of Deformed Microvessels in the Brain in Alzheimer's Disease. Annals of New York Acadmy Sciences, 26, 75-91. https://www.ncbi.nlm.nih.gov/pubmed/9329682?dopt=Abstract\%20

[16] Kalaria, R. (2002) Small Vessel Disease and Alzheimer's Dementia: Pathological Considerations. Cerebrovascular Diseases, 13, 48-52. https://doi.org/10.1159/000049150

[17] Maksimovich, I.V. (2011) Dyscirculatory Angiopathy of Alzheimer's Type. Journal of Behavioral and Brain Science, 1, 57-68. https://doi.org/10.4236/jbbs.2011.12008

[18] Zlokovic, B.V. (2011) Neurovascular Pathways to Neurodegeneration in Alzheimer's Disease and Other Disorders. Nature Reviews. Neuroscience, 3, 723-738. https://doi.org/10.1038/nrn3114

[19] Maksimovich, I.V. (2012) Vascular Factors in Alzheimer's Disease. Health, 4, 735-742. http://file.scirp.org/Html/8-8201699_23274.htm https://doi.org/10.4236/health.2012.429114

[20] Baloiannis, S.J. and Baloiannis, I.S. (2012) The Vascular Factor in Alzheimer's Disease: A Study in Golgi Technique and Electron Microscopy. Journal of the Neurological Sciences, 322, 117-121. https://doi.org/10.1016/j.jns.2012.07.010

[21] Baloyannis, S.J. (2015) Brain Capillaries in Alzheimer's Disease. Hellenic Journal of Nuclear Medicine, 18, 152.http://www.nuclmed.gr/magazine/eng/sept15/suppl.pdf https://doi.org/10.15406/jnsk.2015.02.00069

[22] Iadecola, C. (2004) Neurovascular Regulation in the Normal Brain and in Alzheimer's Disease. Nature Reviews Neuroscience, 5, 347-360. https://doi.org/10.1038/nrn1387

[23] Maksimovich, I.V. and Gotman, L.N. (2006) Method of Complex Radiation Diagnostics at Preclinical and Clinical Stages of Alzheimer's Disease. Russian Patent No. 2315559.

https://worldwide.espacenet.com/publicationDetails/biblio?II=3\&ND=3\&adjacent= 
true\&locale $=$ en_EP\&FT $=$ D\&date $=20080127 \& C C=R U \& N R=2315559 \mathrm{C} 1 \& \mathrm{KC}=\mathrm{C} 1 \#$

[24] Maksimovich, I.V., Gotmanm, L.N. and Masyuk, S.M. (2006) Method of Determining Dimensions of Temporal Brain Lobes in Patients Suffering from Alzheimer's Disease. Russian Patent No. 2306102.

https://worldwide.espacenet.com/publicationDetails/biblio?DB=EPODOC\&adjacent= true\&locale $=$ en_EP\&FT $=$ D\&date $=20070920 \& C C=R U \& N R=2306102 C 1 \& K C=C 1 \#$

[25] Bell, R.D. and Zlokovic, B.V. (2009) Neurovascular Mechanisms and Blood-Brain Barrier Disorder in Alzheimer's Disease. Acta Neuropathologica, 118, 103-113. https://doi.org/10.1007/s00401-009-0522-3

[26] Koike, M.A., Green, K.N. and Blurton-Jones, M. (2010) Oligemic Hypoperfusion Differentially Affects Tau and Amyloid-\{Beta\}. The American Journal of Pathology, 177, 300-310. https://doi.org/10.2353/ajpath.2010.090750

[27] Nelson, A.R., Sweeney, M.D., Sagare, A.P. and Zlokovic, B.V. (2016) Neurovascular Dysfunction and Neurodegeneration in Dementia and Alzheimer's Disease. Biochimica et Biophysica Acta, 1862, 887-900. https://doi.org/10.1016/j.bbadis.2015.12.016

[28] Kimbrough, I.F., Robel, S., Roberson, E.D. and Sontheimer, H. (2015) Vascular Amyloidosis Impairs the Gliovascular Unit in a Mouse Model of Alzheimer's Disease. Brain, 138, 3716-3733. https://doi.org/10.1093/brain/awv327

[29] Maksimovich, I.V. (2008) Radiodiagnostics of Alzheimer's Disease. Diagnostics and Intervention Radiology, 4, 27-38. http://radiology-diagnos.ru/articles/2008-4-04

[30] Morris, J.C. (1993) The Clinical Dementia Rating (CDR): Current Version and Scoring Rules. Neurology, 11, 2412-2414. https://www.ncbi.nlm.nih.gov/pubmed/8232972 https://doi.org/10.1212/WNL.43.11.2412-a

[31] Folstein, M.F., Folstein, S.E. and McHugh, P.R. (1975) Mini-Mental State. A Practical Method for Grading the Cognitive State of Patients for the Clinician. Journal of Psychiatric Research, 12, 189-198. https://doi.org/10.1016/0022-3956(75)90026-6 https://www.ncbi.nlm.nih.gov/pubmed/1202204

[32] Maksimovich, I.V. (2012) The Tomography Dementia Rating Scale (TDR)-The Rating Scale of Alzheimer's Disease Stages. Health, 4, 712-719. https://doi.org/10.4236/health.2012.429111

[33] De Lin, M. and Jackson, E.F. (2012) Applications of Imaging Technology in Radiation Research. Radiation Research, 177, 387-397.

https://www.ncbi.nlm.nih.gov/pmc/articles/PMC3355534/ https://doi.org/10.1667/RR2696.1

[34] Brown, W.R. and Thore, C.R. (2011) Review: Cerebral Microvascular Pathology in Ageing and Neurodegeneration. Neuropathology and Applied Neurobiology, 37, 56-74. https://doi.org/10.1111/j.1365-2990.2010.01139.x

[35] Henry-Feugeas, M.C. (2008) Alzheimer's Disease in Late-Life Dementia: A Minor Toxic Consequence of Devastating Cerebrovascular Dysfunction. Medical Hypotheses, 70, 866-875. https://doi.org/10.1016/j.mehy.2007.07.027

[36] Maksimovich, I.V. (2012) Certain New Aspects of Etiology and Pathogenesis of Alzheimer's Disease. Advances in Alzheimer's Disease, 1, 68-76. https://doi.org/10.4236/aad.2012.13009

[37] Maksimovich, I.V. (2013) Disorders of Cerebrovascular Angioarchitectonics and Microcirculation in the Etiology and Pathogenesis of Alzheimer's Disease Advances in Alzheimer's Disease, 2, 171-181. https://doi.org/10.4236/aad.2013.24022 
[38] Zlokovic, B.V. (2010) Neurodegeneration and the Neurovascular Unit. Nature Medicine, 16, 1370-1371. https://www.nature.com/articles/nm1210-1370 https://doi.org/10.1038/nm1210-1370

[39] Iadecola, C. (2010) The Overlap between Neurodegenerative and Vascular Factors in the Pathogenesis of Dementia. Acta Neuropathologica, 120, 287-396. https://doi.org/10.1007/s00401-010-0718-6

[40] De la Torre, J.C. (2012) A Turning Point for Alzheimer's Disease? Bio Factors, 38, 78-83. https://doi.org/10.1002/biof.200

[41] De la Torre, J.C. (2016) Cerebral Perfusion Enhancing Interventions: A New Strategy for the Prevention of Alzheimer Dementia. Brain Pathology, 26, 618-631. https://doi.org/10.1111/bpa.12405

[42] Love, S. and Miners, J.S. (2016) Cerebral Hypoperfusion and the Energy Deficit in Alzheimer's Disease. Brain Pathology, 26, 607-617. https://doi.org/10.1111/bpa.12401

[43] Nielsen, R.B., Egefjord, L., Angleys, H., et al. (2017) Capillary Dysfunction Is Associated with Symptom Severity and Neurodegeneration in Alzheimer's Disease. Alzheimer's \& Dementia, 13, 1143-1153. https://doi.org/10.1016/j.jalz.2017.02.007

[44] Bosco, P., Redolfi, A., Bocchetta, M., et al. (2017) The Impact of Automated Hippocampal Volumetry on Diagnostic Confidence in Patients with Suspected Alzheimer's Disease: An EADC Study. Alzheimer's \& Dementia, 13, 1013-1023. https://doi.org/10.1016/j.jalz.2017.01.019

[45] Iadecola, C. (2015) Dangerous Leaks: Blood-Brain Barrier Woes in the Aging Hippocampus. Neuron, 85, 231-233. https://doi.org/10.1016/j.neuron.2014.12.056

[46] Montagne, A., Barnes, S.R., Sweeney, M.D., et al. (2015) Blood-Brain Barrier Breakdown in the Aging Human Hippocampus. Neuron, 85, 296-302. https://doi.org/10.1016/j.neuron.2014.12.032

[47] Maksimovich, I.V. (2017) Morphometric Definition of Alzheimer's Disease Stages by Means of the Tomography Dementia Rating Scale (TDR). Brain Disorders and Therapy, 6, 1-4. https://doi.org/10.4172/2168-975X.1000238

[48] Schmidtke, K. and Hull, M. (2005) Cerebral Small Vessel Disease: How Does It Progress? Journal of the Neurological Sciences, 229-230, 13-20. https://doi.org/10.1016/j.jns.2004.11.048

[49] Qureshi, A.I. and Caplan, L.R. (2014) Intracranial Atherosclerosis. The Lancet, 15, 984-998. https://doi.org/10.1016/S0140-6736(13)61088-0

[50] Caplan, L.R. (2016) The Effect of Small Artery Disease on the Occurrence and Management of Large Artery Disease. JAMA Neurology, 73, 19-20. https://doi.org/10.1001/jamaneurol.2015.3535

[51] Ramos-Estebanez, C., Moral-Arce, I., Gonzalez-Mandly, A., et al. (2011) Vascular Cognitive Impairment in Small Vessel Disease: Clinical and Neuropsychological Features of Lacunar State and Binswanger's Disease. Age Ageing, 40, 175-180. https://doi.org/10.1093/ageing/afq169

[52] Akiguchi, I., Budka, H., Shirakashi, Y., et al. (2014) MRI Features of Binswanger's Disease Predict Prognosis and Associated Pathology. Annals of Clinical and Translational Neurology, 1, 813-821. https://doi.org/10.1002/acn3.123

[53] Maksimovich, I.V. (2017) Possibilities of Application of Transcatheter Treatment of Vascular Dementia with Binswanger's Disease. Global Journal of Health Science, 9, 13-21. https://doi.org/10.5539/gjhs.v9n6p13 